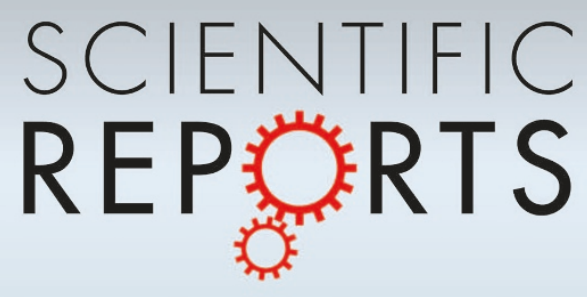

OPEN

SUBJECT AREAS:

FERROELECTRICS AND

MULTIFERROICS

ELECTROCATALYSIS

ELECTROCHEMISTRY

SURFACES, INTERFACES AND

THIN FILMS

Received

25 April 2013

Accepted

20 June 2013

Published

8 July 2013

Correspondence and requests for materials should be addressed to X.W. (xudong@engr. wisc.edu)

\section{Fundamental Analysis of Piezocatalysis Process on the Surfaces of Strained Piezoelectric Materials}

\author{
Matthew B. Starr \& Xudong Wang
}

Department of Materials Science and Engineering, University of Wisconsin-Madison,

Recently, the strain state of a piezoelectric electrode has been found to impact the electrochemical activity taking place between the piezoelectric material and its solution environment. This effect, dubbed piezocatalysis, is prominent in piezoelectric materials because the strain state and electronic state of these materials are strongly coupled. Herein we develop a general theoretical analysis of the piezocatalysis process utilizing well-established piezoelectric, semiconductor, molecular orbital and electrochemistry frameworks. The analysis shows good agreement with experimental results, reproducing the time-dependent voltage drop and $\mathrm{H}_{2}$ production behaviors of an oscillating piezoelectric $\mathrm{Pb}\left(\mathrm{Mg}_{1 / 3} \mathrm{Nb}_{2 / 3}\right) \mathrm{O}_{3}-32 \mathrm{PbTiO}_{3}(\mathrm{PMN}-\mathrm{PT})$ cantilever in deionized water environment. This study provides general guidance for future experiments utilizing different piezoelectric materials, such as $\mathrm{ZnO}, \mathrm{BaTiO}_{3}, \mathrm{PbTiO}_{3}$, and $\mathrm{PMN}-\mathrm{PT}$. Our analysis indicates a high piezoelectric coupling coefficient and a low electrical conductivity are desired for enabling high electrochemical activity; whereas electrical permittivity must be optimized to balance piezoelectric and capacitive effects.

iezocatalysis is a new approach toward enabling or enhancing electrochemical processes by making use of the strain state of a piezoelectric material ${ }^{1}$. Piezocatalysis is the product of an intimate interaction between the native electronic state of the piezoelectric material, the chemistry of the surrounding medium, and a strain induced piezoelectric potential. The action of mechanically deforming a piezoelectric material induces a perfuse electric field which augments the energetics of both free and bound charges throughout the material ${ }^{1,2}$. The thermodynamic feasibility and kinetics of electrochemical processes occurring at the surface of the piezoelectric material sensitively depend upon the electrochemical potential difference between charges on the piezoelectric's surface and in the surrounding medium ${ }^{3-6}$. Thus piezoelectric potential, which can dramatically affect the difference between these electrochemical potentials, is a new means of modulating the material's electrochemical activity via its strain state.

Recently, a piezocatalysis process was demonstrated by our study of a strained ferroelectric $\mathrm{Pb}\left(\mathrm{Mg}_{1 / 3} \mathrm{Nb}_{2 / 3}\right) \mathrm{O}_{3^{-}}$ $32 \mathrm{PbTiO}_{3}$ (PMN-PT) beam in a deionized (DI) water system, from which a strong dependence of hydrogen evolution from the aqueous surroundings on the material's piezoelectric potential was observed ${ }^{1}$. In addition, numerous recent works have confirmed the correlation between electrochemical activity and piezoelectric or ferroelectric polarization in a broader sense. For example, a study conducted using ferroelectric poly(vinylidene fluoride) (PVDF) has demonstrate that in-situ piezopotential can influence lithium battery charging behavior? Electrochemical deposition was found to be selectively activated by the ferroelectric domain polarization ${ }^{8-15}$. However, to date there is no general theoretical analysis of the piezopotential's effect on electrochemical activities, e.g. how one piezoelectric material's activity differs from another; the influence of metallic electrodes as compared to bare piezoelectric surface on reaction output, and how free charge in piezoelectric material systems affects the piezocatalysis process. In this paper, we address the piezocatalysis system in generality to elucidate the details underlying these open questions and illuminate trends for further experimental study.

In order to clearly understand the piezocatalysis process, a conventional electrocatalysis process is discussed first, where the application of electrical potential from an external power source is a typical means of driving the electron transfer reactions (Fig. 1a). The applied potential can result in one of the following two processes: (1) lowering electronic energy levels of unoccupied states within the electrode to a magnitude less than that of the highest occupied molecular orbital (HOMO) in solution; (2) raising occupied states within the electrode above the lowest unoccupied molecular orbital (LUMO) in solution. Under the first condition, electrons will leave the HOMOs in solution and transfer to the unoccupied states within the electrode - oxidizing the solution 
a

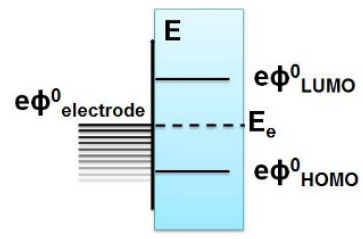

b Energy
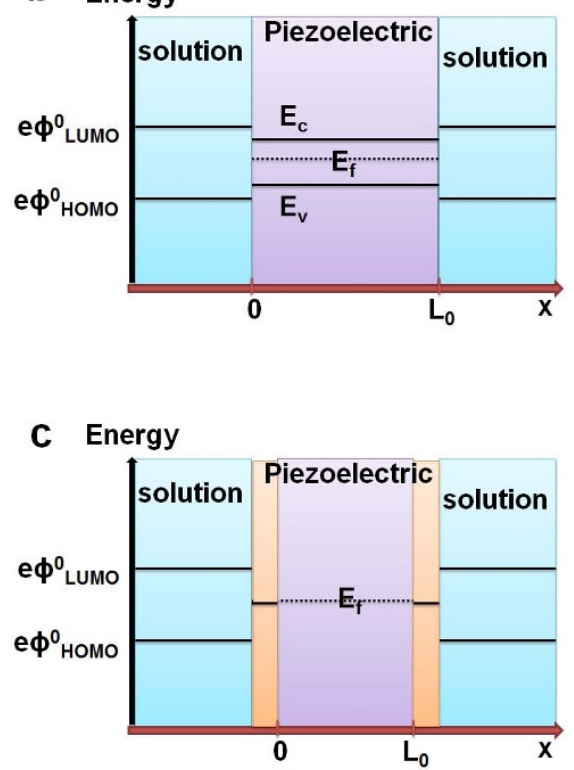
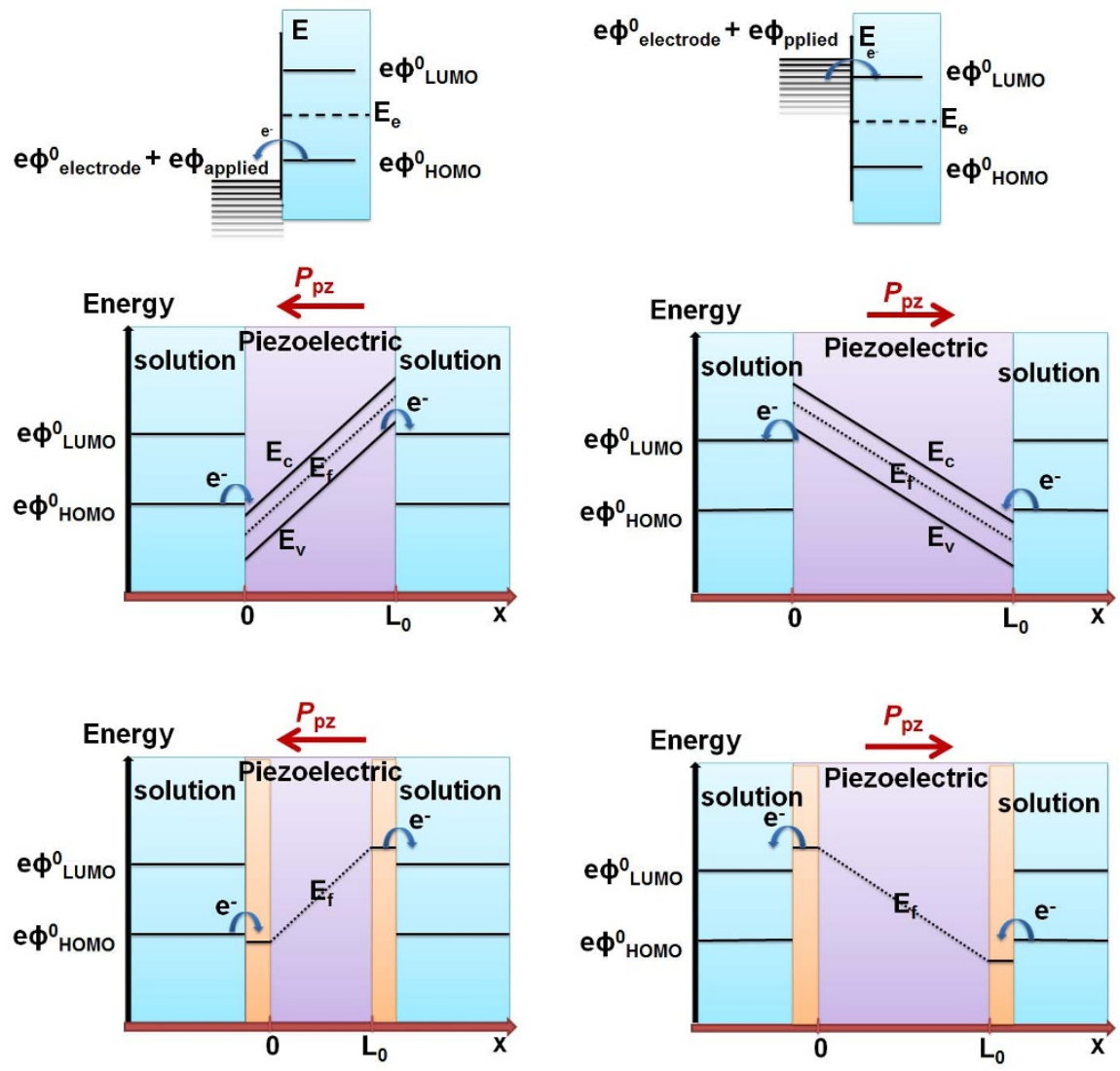

Figure 1 Energy diagrams describing the electrochemistry and piezocatalysis process. (a) The effect of applying a sufficient positive or negative bias to an electrode is to increase anodic and cathodic current, respectively. (b) In the case of a bare piezoelectric, conduction and valence bands act as the reservoirs for electrons donated or accepted from molecules in solution. The piezoelectric polarization $\left(P_{\mathrm{PZ}}\right)$ applies a variable bias across the material, lifting and lowering valence band and conduction band energies. (c) Applying electrodes to the piezoelectric simplifies the electron reservoir to that of the metal's Fermi energy, while maintaining the piezoelectric potential as the source of bias.

(center panel of Fig. 1a). Under the second condition, electrons will leave the occupied states in the electrode and transfer to the LUMOs in solution - reducing the solution (right panel of Fig. 1a). When the electrode is a non-metallic solid, the e $\varphi_{\text {HOMO }}$ and $\mathrm{e} \phi_{L U M O}$ logic extends to the materials valence $\left(\mathrm{e} \varphi_{\mathrm{VB}}\right)$ and conduction band $\left(\mathrm{e} \varphi_{\mathrm{CB}}\right)$ edges, respectively.

For piezocatalysis, the external power source is replaced by piezoelectric potential which results from the piezoelectric polarization $\left(P_{\mathrm{PZ}}\right)$. A perfectly insulating piezoelectric material is the most ideal and simplest case and it will be analyzed first. For such a material, mechanical deformation creates a perfuse electric field inducing a total energy shift across the material, $\mathrm{V}_{\mathrm{p}_{\text {max }}}$, given by:

$$
\mathrm{V}_{\mathrm{p}_{\text {max }}}=\frac{\mathrm{w}_{x} \mathrm{~T}_{\mathrm{k}} \mathrm{d}_{x k}}{\varepsilon_{0} \varepsilon_{r, x}}
$$

where $\mathrm{T}_{\mathrm{k}}$ is an applied stress in the $k$ dimension, $\mathrm{d}_{x k}$ are the piezoelectric moduli, $\varepsilon_{0}$ is the electrical permittivity of free space, and $\varepsilon_{r, x}$ is the relative permittivity in the $\mathrm{x}$ dimension and $\mathrm{W}_{x}$ is the width of the piezoelectric material in the $x$ dimension. In a one-dimensional case, $x$ and $k$ are equal and the subscripts are dropped.

This piezoelectric potential, which changes the energetics of the valence band (VB) and conduction band (CB) across the piezoelectric material, can have a dramatic effect on the material's interaction with its environment (Fig. 1b). In this model it is assumed that stain does not change the magnitude of the band gap. If the $\mathrm{e} \varphi_{\mathrm{VB}}$ approaches e $\phi_{L U M O}$, it becomes energetically favorable for electrons to leave the VB and enter the LUMO (center panel of Fig. 1b). If the $\mathrm{e} \varphi_{\mathrm{CB}}$ approaches $\mathrm{e} \varphi_{\mathrm{HOMO}}$, it becomes energetically favorable for electrons to leave the HOMO and enter the CB (right panel of Fig. 1b). Placing metal electrodes between the piezoelectric and solution, with their continuous density of states about their Femi energies, simplifies this situation: the piezopotential now acts as a bias, lifting and lowering the metal's Fermi energy e $\varphi_{M}$ (Fig. 1c).

Piezoelectric materials, which are the source of bias in the case of piezocatalysis, are capable of achieving extremely high potentials (tens to hundreds of volts) when subjected to moderate to severe strain $^{16}$. Under such circumstances (i.e. where electrode potentials versus standard hydrogen electrode (SHE) exceeding $\sim 3$ volts) many chemical species in contact with the piezoelectric will be thermodynamically capable of undergoing reduction or oxidation reactions.

In order to deduce the maximum quantity of oxidation or reduction reactions possible for a given deformation, it is necessary to quantify the interaction between charge exchange at the piezoelectric/electrolyte interface and the effect of charge exchange on the piezopotential. A characteristic of piezocatalytic systems is the piezopotential drop that takes place during charge transfer to and from its surfaces (or electrodes). In this way the system acts as a capacitor. The rate of piezopotential drop is dependent upon both the piezoelectric material's properties and the nature of the solution in which it is submerged. In the case where the concentration of reactive species in solution is low, e.g. when pure Milli-Q water is used for the hydrogen reduction reaction, and the power available for driving electron-transfer reactions is sufficiently high, the rate of electrochemical reactions at the electrode is dominated by the diffusion rate of redox species to the electrode. In the case of Milli-Q water, the redox species are protons, hydroxide and impurity species. Mass transfer to the electrode is described by the Nerst-Planck 
equation, which in its one-dimensional form along the $\mathrm{x}$-axis is written as:

$$
\mathrm{J}_{\mathrm{i}}(\mathrm{x})=-\mathrm{D}_{\mathrm{i}} \frac{\partial \mathrm{C}_{\mathrm{i}}(\mathrm{x})}{\partial x}-\frac{\mathrm{F}}{\mathrm{RT}} \mathrm{D}_{\mathrm{i}} \mathrm{C}_{\mathrm{i}}(\mathrm{x}) \frac{\partial \varphi(x)}{\partial x}+\mathrm{C}_{\mathrm{i}} v(\mathrm{x})
$$

where $\mathrm{J}_{\mathrm{i}}(\mathrm{x})$ is the flux of species $i$ at distance $x$ from the surface, $\mathrm{D}_{\mathrm{i}}$ is the diffusion coefficient, $\frac{\partial \mathrm{C}_{\mathrm{i}}(\mathrm{x})}{\partial x}$ is the concentration gradient at distance $\mathrm{x}, F$ is the Faraday constant, $R$ is the gas constant, $T$ is absolute temperature, $\frac{\partial \varphi(x)}{\partial x}$ is the potential gradient, $\mathrm{C}_{\mathrm{i}}$ is the concentration of species $i$, and $v(\mathrm{x})$ is the velocity with which a volume element in solution moves along the axis ${ }^{17}$. The expression describes the contributions of diffusion, migration and convention, respectively, to the flux of species $i$.

Neglecting migration and convective phenomena, the flux of species $i$ is dependent upon diffusion alone. In the diffusion limited regime, the maximum rate of electron transfer from the piezoelectric is equal to the rate of reactant diffusion to the piezoelectric, resulting in a current density $j$ given by:

$$
j_{\mathrm{i}}(\mathrm{x})=-\mathrm{n}_{\mathrm{i}} \mathrm{FD}_{\mathrm{i}} \frac{\partial \mathrm{C}_{\mathrm{i}}(\mathrm{x})}{\partial x}
$$

where $\mathrm{n}_{\mathrm{i}}$ is the number of electrons per reaction event with species $i^{17}$.

Applying appropriate boundary conditions to equation (3) and taking account of the capacitive nature of the piezoelectric bias source, yields an expression of the piezopotential $V_{\mathrm{p}}$ as a function of time (a variant on the Cottrell equation):

a
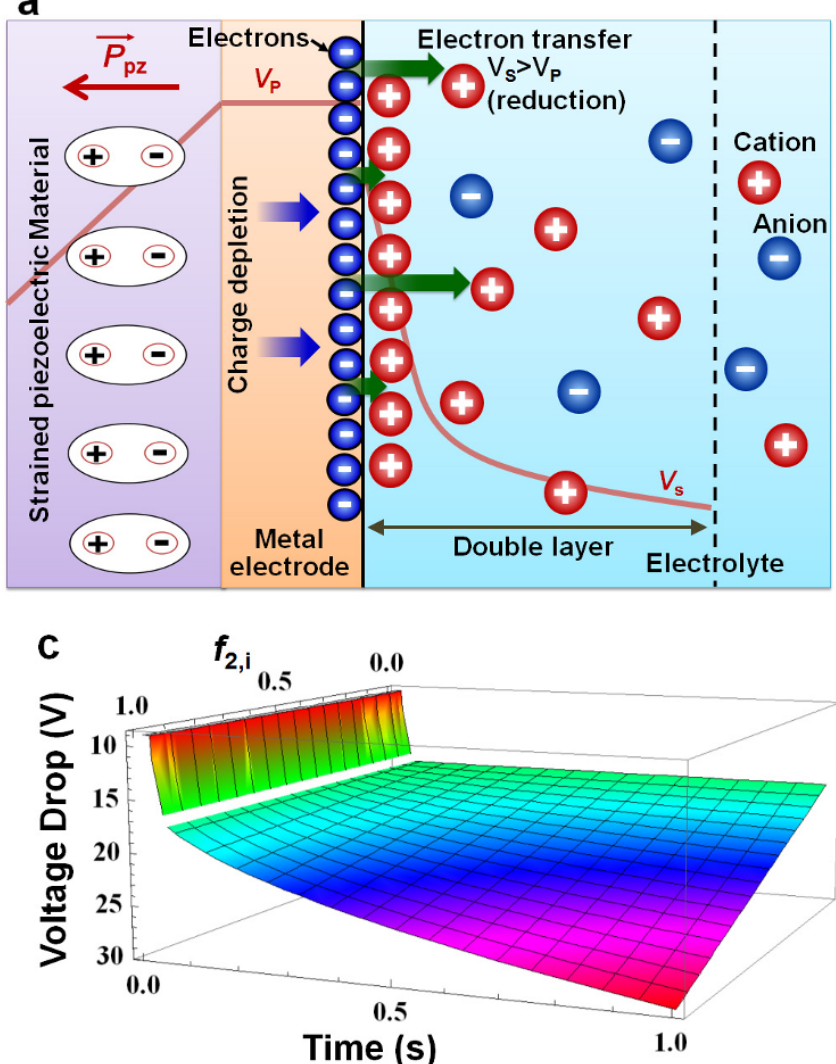

$$
\frac{\partial V_{p}}{\partial t}=-\frac{w_{x} F \sum n_{i} D_{i}{ }^{1 / 2} c_{i} f_{i}}{t^{1 / 2} \varepsilon_{0} \varepsilon_{r, x} \pi^{1 / 2}}
$$

where $c_{\mathrm{i}}$ is the bulk concentration of species $i, f_{\mathrm{i}}$ is a parameter taking on a value between 0 and 1 that toggles the kinetics of the electrodes' reactivates with species $i$, and $t$ is time ${ }^{18}$.

Under conditions of low reactant and electrolyte concentrations and high positive electrode potential (e $\phi_{L U M O}>2 \mathrm{eV}$ vs SHE), a kinetics parameter $\left(f_{\mathrm{i}}\right)$ less than 1 causes a semi-diffusion controlled regime to form where charged reactant species (e.g. protons and metal ions) capactively couple to the electrode's surface, effectively reducing the surface potential before they are electrochemically reduced or oxidized (Fig. 2a). Under the application of a large positive electrode potential, a dilute (e.g. Milli-Q water) system's capacitance is well approximated by the Helmholtz model ${ }^{17}$. The voltage drop expected in time $t$ by both electron transfer reactions and capacitive effects is given by integrating equation (4):

$V_{p}=-\frac{2 F t^{1 / 2}}{\varepsilon_{0} \varepsilon_{r, x} \pi^{1 / 2}}\left[w_{x} \sum_{i=l}^{i=1} n_{i} D_{i}{ }^{1 / 2} c_{i} f_{i}+w_{H} \sum_{i=l}^{i=1} z_{i} n_{i} D_{i}{ }^{1 / 2} c_{i}\left(1-f_{i}\right)\right]$

where $z_{\mathrm{i}}$ is the charge sign ( 1 for cation, -1 for anion) of species $i, w_{H}$ is the thickness of the Helmholtz layer, and $l$ is the number of different species in solution. The positive potential of the electrode dictates that a positive charge in solution will contribute to a positive capacitive current, lowering the piezopotential. The first term in equation (5) describes the piezopotential change as redox reactions proceed, the second term describes the potential change due

b

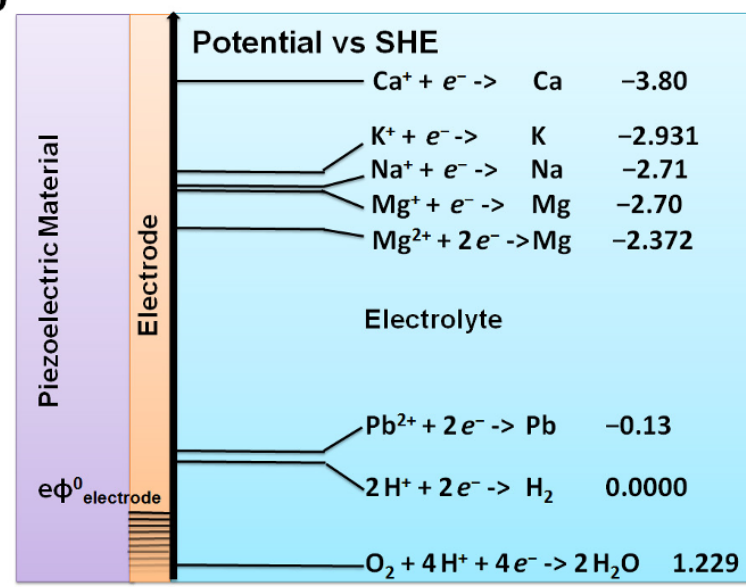

d

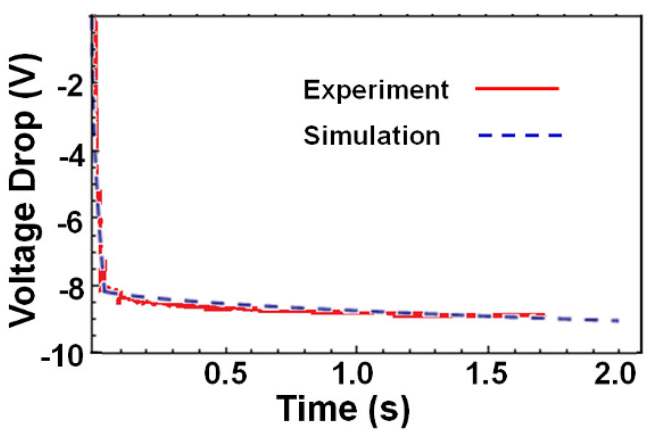

Figure $2 \mid$ The voltage drop across a strained piezoelectric is dependent upon the rate of capacitive and Faradic effects. (a) The charged piezoelectric's surface induces both capacitive double layer effects and electron transfer events across the interface. (b) At high negative piezoelectric potentials, the electrode is thermodynamically capable of reducing all reported impurity ions in solution. As the potential increases below -2.0 volts, the majority of side reactions are no longer possible. (c) Depending on $t_{p}$ and the values of kinetic parameters $f_{i, 1}$ and $f_{i, 2}$, the rate of voltage drop experienced as a function of time can take on a wide range of values. (d) Applying a recursion procedure to the data obtained by Wang et al, values of $\mathrm{t}_{\mathrm{p}}=0.042 \mathrm{~s}$, $\mathrm{f}_{\mathrm{i}, 1}=0.715$ and $\mathrm{f}_{\mathrm{i}, 2}=0.07$ are determined. 
to capacitive coupling of not yet reacted species at the piezoelectric material's surface.

Equation (5) governs how the potential on the piezoelectric's wall should drop from the time $(t=0)$ of initial mechanical deformation, when the piezopotential can be a value of tens or hundreds of volts, to a time $\left(t=t_{\mathrm{p}}\right)$ when the potential has been reduced to a reasonable value about the SHE (e.g. $\pm 2 \mathrm{~V}$ vs SHE). Between time $0<t<t_{\mathrm{p}}$, the electrode potential is sufficiently high as to reduce or oxidize species within solution in an effectively nonselective manner. At $t>t_{\mathrm{p}}$, the piezoelectric potential falls within the range of typical $е \varphi_{\text {Номо }}$ and e $\phi_{L U M O}$ values of ions in solution resulting in a more selective reduction and oxidation process (Fig. 2b).

The processes described above pertain to both surfaces of the piezoelectric material; on one surface oxidation dominates while reduction dominates on the other. The full voltage change, as measured across the piezoelectric material, is a combination of both of these processes happening simultaneously. An electrode potential above (in the case of oxidation current) or below (reduction current) the selective potential window of $\sim \pm 2 \mathrm{~V}$ versus SHE here are treated identically. This results in a total voltage reduction across the piezoelectric material of twice the expected magnitude from a single wall (equation (5)).

As the piezopotential reaches a modest range about $0 \mathrm{~V}\left(t=t_{\mathrm{p}}\right)$, the electrochemical activity of some ions is effectively switched off while others will remain highly active. Those ions which are no longer electrochemically active are still free to participate in capacitive effects (Fig. $2 \mathrm{~b}$ and second term in equation (5)). The voltage decrease across the piezoelectric from $t_{\mathrm{p}} \geq t \geq \infty$ is thus due to a separate set of electrochemical and capacitive effects conducted by a subset (i...m) of the total chemical species present (i...l). By applying equation (5) to both the selective and unselective regime we can describe the magnitude of piezopotential at all times by the following derived expression:

$$
\begin{aligned}
V_{p}= & -H\left(t_{p}-t\right)\left(\frac { 4 F t ^ { 1 / 2 } } { \varepsilon _ { 0 } \pi ^ { 1 / 2 } } \left[\frac{w_{x}}{\varepsilon_{r, x}} \sum_{i=l}^{i=1} n_{i} D_{i}{ }^{1 / 2} c_{i} f_{1, i}+\right.\right. \\
& \left.\left.\frac{w_{H}}{\varepsilon_{H}} \sum_{i=l}^{i=1} z_{i} n_{i} D_{i}{ }^{1 / 2} c_{i}\left(1-f_{1, i}\right)\right]\right)- \\
& H\left(t-t_{p}\right)\left(\frac { 4 F t ^ { 1 / 2 } } { \varepsilon _ { 0 } \pi ^ { 1 / 2 } } \left[\frac{w_{x}}{\varepsilon_{r, x}} \sum_{i=m}^{i=1} n_{i} D_{i}{ }^{1 / 2} c_{i} f_{2, i}+\right.\right. \\
& \left.\frac{w_{H}}{\varepsilon_{H}} \sum_{i=m}^{i=1} z_{i} n_{i} D_{i}{ }^{1 / 2} c_{i}\left(1-f_{2, i}\right)+\frac{w_{H}}{\varepsilon_{H}} \sum_{i=l}^{i=m+1} z_{i} n_{i} D_{i}{ }^{1 / 2} c_{i}\right]+ \\
& \left(\frac{4 F t^{1 / 2}}{\varepsilon_{0} \pi^{1 / 2}}\left[\frac{w_{x}}{\varepsilon_{r, x}} \sum_{i=l}^{i=1} n_{i} D_{i}{ }^{1 / 2} c_{i} f_{1, i}+\frac{w_{H}}{\varepsilon_{H}} \sum_{i=l}^{i=1} z_{i} n_{i} D_{i}{ }^{1 / 2} c_{i}\left(1-f_{1, i}\right)\right]-\right. \\
& \left(\frac { 4 F t ^ { 1 / 2 } } { \varepsilon _ { 0 } \pi ^ { 1 / 2 } } \left[\frac{w_{x}}{\varepsilon_{r, x}} \sum_{i=m}^{i=1} n_{i} D_{i}{ }^{1 / 2} c_{i} f_{2, i}+\right.\right. \\
& \left.\left.\left.\frac{w_{H}}{\varepsilon_{H}} \sum_{i=m}^{i=1} z_{i} n_{i} D_{i}{ }^{1 / 2} c_{i}\left(1-f_{2, i}\right)+\frac{w_{H}}{\varepsilon_{H}} \sum_{i=l}^{i=m+1} z_{i} n_{i} D_{i}{ }^{1 / 2} c_{i}\right]\right)\right)
\end{aligned}
$$

where $H(\mathrm{x})$ is the Heaviside function defined as 0 when $x$ is less than 0 , and 1 when $x$ is equal to or greater than $0 . \varepsilon_{H}$ is the electrical permittivity of the Helmholtz layer. The third and fourth terms in equation (6) acts to add continuity between the selective and unselective regimes.

Equation (6) is a simplification of the activity occurring around the piezoelectric as the piezopotential drops to within the vicinity of $\mathrm{e} \varphi_{\text {HOMO }}$ and e $\phi_{\text {LUMO }}$ of species in solution. As the energetic advantage for electron transfer diminishes, the current density $j$ begins to show its exponential dependence upon driving force $\left(\mathrm{e} \varphi_{\text {HOMO }}-\mathrm{e} \phi_{L U M O}\right)$. The current density exponentially depends upon applied potential about $E_{\mathrm{e}}$ :

$$
j=j_{0}\left(\exp ^{\frac{\alpha_{a} n F \eta}{R T}}-\exp ^{\frac{\left(1-\alpha_{a}\right) n F \eta}{R T}}\right)
$$

where $j_{0}$ is the exchange current density and is equal to:

$$
j_{0}=n F k_{a}^{0} c_{R} \exp ^{\frac{\alpha_{a} n F E_{e}}{R T}}=n F k_{C}^{0} c_{O} \exp ^{\frac{\left(1-\alpha_{a}\right) n F E_{e}}{R T}}
$$

$n$ is the number of electrons transferred in the reaction, $k_{a}^{0}$ and $k_{C}^{0}$ are the rate constants for the anodic and cathodic current, respectively, $C_{\mathrm{R}}$ and $C_{\mathrm{O}}$ are the concentrations of the reduced and oxidized species, respectively, $\alpha_{a}$ is the anodic transfer coefficient, $E_{\mathrm{e}}$ is the redox potential of a species in solution, and $\eta$ is the applied bias (i.e. Fermi energy difference between $e \varphi_{m}$ and $\left.E_{\mathrm{e}}\right)^{18}$. For the purposes of simplification, the effects of equation (7) will be approximated by utilizing two different kinetics constants acting in equation (6) for $1 \geq i \geq m$ : $f_{1, \mathrm{i}}$ and $f_{2, \mathrm{i}}$ for the nonselective time interval $0 \leq t \leq t_{\mathrm{p}}$ and the selective interval $t_{\mathrm{p}} \geq t \geq \infty$, respectively.

To elucidate the behavior of the system, Fig. $2 \mathrm{c}$ is a plot of equation (6) as it is applied to the case of a piezoelectric material (PMN-PT) strained in Milli-Q water. We take a value of electrical permittivity $\varepsilon_{P M N-P T, x}=8000$ and a thickness of PMN-PT $w_{\text {th }}=.23 \mathrm{~mm}^{19}$. The value of the Helmhotlz layer thickness $\left(w_{H}\right)$ was fixed to $2.75 \AA$ with a $\varepsilon_{H}=80$. Under standard conditions, Milli-Q water has a resistivity value of $18 \Omega \cdot \mathrm{cm}$ due to the contribution of $l$ "impurities", including hydroxide and hydronium $\left(c_{1,2}=\sim 0.100 \mathrm{ppb} \cdot \mathrm{mol} \cdot \mathrm{cm}^{-3}, D_{1,2}=\right.$ $\left.9.3 * 10^{-5} \mathrm{~cm}^{2} \mathrm{sec}\right)$, organics $\left(c_{3}=1.106 \mathrm{ppb} \cdot \mathrm{mol} \cdot \mathrm{cm}^{-3}, D_{3}=0.52 *\right.$ $\left.10^{-5} \mathrm{~cm}^{2} \mathrm{sec}\right)$, silicates $\left(c_{4}=0.5534 \mathrm{ppb} \cdot \mathrm{mol} \cdot \mathrm{cm}^{-3}, D_{4}=1.28 *\right.$ $10^{-5} \mathrm{~cm}^{2} \mathrm{sec}$ ), and heavy metal ions (calcium, sodium, chloride, etc; $\left.c_{5}=0.1106 \mathrm{ppb} \cdot \mathrm{mol} \cdot \mathrm{cm}^{-3}, D_{5}=1.2 * 10^{-5} \mathrm{~cm}^{2} \mathrm{sec}\right)^{20}$. Based on experimental measurements discussed in the following section, $t_{p}$ is chosen at $0.042 \mathrm{~s}$ and $f_{1 i}$ is 0.715 (between $0 \geq t>t_{p}, 71.5 \%$ of all species immediately undergo redox reactions upon their contact with the electrode, $28.5 \%$ of species first capacitively couple to the electrode).

The physical consequences of the voltage decrease described by equation (6) are: (1) the creation of reaction byproducts in the medium surrounding the piezoelectric material (e.g. hydrogen gas, oxygen gas, chlorine gas, oxidized organics, etc); (2) the buildup of a capacitive layer in solution alongside the piezoelectric material; and (3) a reduction in the electric field present inside the piezoelectric material. These effects will continue to change with time until the potential decrease (equation (6)) approaches the total potential originally generated (equation (1)). The rate of these effects depend sensitively upon the rate constants $f_{1 i}$ and $f_{2 i}$, the concentration of reactive species and their diffusion coefficients. The natural driving force for these chemical changes is the piezopotential which, as described by equation (1), depends upon the electrical permittivity, mechanical strain, Young's moduli and piezoelectric moduli. This is true regardless of whether the electrochemically active medium is in contact with metallic electrodes or the piezoelectric material's bare surfaces. In the case of bare piezoelectric surfaces, the band gap and positions of $\mathrm{e} \phi_{C B}$ and $\mathrm{e} \phi_{V B}$ relative to $\mathrm{e} \varphi_{\mathrm{HOMO}}$ and $\mathrm{e} \phi_{L U M O}$ also determine the nature of what happens during straining.

When comparing the piezocatalytic abilities of various piezoelectric materials, it is useful to choose a performance metric from equation (6) such as the quantity of a reaction byproduct, e.g. $\mathrm{H}_{2}$ gas. This value depends sensitively upon the parameters we choose. Previous work reported the production of hydrogen gas via piezocatalysis between a PMN-PT single crystal slab $(2 \mathrm{~mm} \times 10 \mathrm{~mm}$ in size) and Milli-Q water ${ }^{1}$. Using recursion we are able to fit equation (6) to the piezopotential verses time profile obtained from a working electrode on a PMN-PT surface under one strain (Fig. 2d). The results yield $t_{\mathrm{p}}=0.042 \mathrm{~s}, f_{1 \mathrm{i}}=0.715$, and $f_{2 \mathrm{i}}=0.07$ under the constraints that after $t=t_{\mathrm{p}}$ only $\mathrm{H}^{+}$reduction and $\mathrm{H}_{2} \mathrm{O}$ oxidation were possible at the electrodes, whilst unreacted $\mathrm{H}^{+}, \mathrm{OH}^{-}$, silicate 
and heavy metal ions only acted capacitively. These low kinetics values likely resulted from a low concentration of electrolyte. The switch over at $t_{\mathrm{p}}=0.042 \mathrm{~s}$ from one reaction regime to the other occurred at a potential (difference) value between the electrodes of approximately $2 \mathrm{~V}$. A gold electrode in equilibrium with $\mathrm{pH} 7 \mathrm{Milli}-$ Q water is in equilibrium with the $\mathrm{H}^{+}$reduction and $\mathrm{H}_{2} \mathrm{O}$ oxidation reactions. This places the Au electrode's potential $\left(\varphi_{\text {ElectrodeHOMO }}\right)$ at $0.41 \mathrm{~V}$ vs. SHE $\left(0.615 \mathrm{~V}\right.$ below the $\varphi_{\text {LUMO }}$ of $\mathrm{H}^{+}$and -0.615 above the e $\varphi_{\mathrm{HOMO}}$ of $\left.\mathrm{H}_{2} \mathrm{O}\right)$. A symmetric voltage shift applied to both $\mathrm{Au}$ electrodes of $1 \mathrm{~V}$ at $t_{p}$ means an overpotential $\left(\varphi_{\text {Electrode HOMO }}-\right.$ $\left.\varphi_{\text {LUMO }}\right)$ of $-0.385 \mathrm{~V}$ for the $\mathrm{H}^{+}$reduction reaction and an overpotential $\left(\varphi_{\text {Electrode HOMO }}-\varphi_{\text {LUMO }}\right)$ of $0.385 \mathrm{~V}$ for the $\mathrm{H}_{2} \mathrm{O}$ oxidation reaction. These are the potential values taken at which the system switches from a nonselective regime to a selective regime.

In order to derive a function of $\mathrm{H}_{2}$ evolution dependency on strain, the minimum strain $S_{\min , 1}$ capable of driving electrochemical reactions for an arbitrary piezoelectric is necessary and determined by augmenting equation (1):

$$
S_{\min , 1}=\frac{\varepsilon_{0} \varepsilon_{r, x}\left(\varphi_{\text {ElectrodeHOMO }}-\varphi_{\mathrm{LUMO}}\right)}{2 w_{x} Y d_{x k}}
$$

where $Y$ is the Young's modulus of the material. For metallic Au electrodes, the preceding analysis determined the potential necessary to drive electrochemical $\mathrm{H}_{2}$ production under the observed conditions to be $0.615\left(\varphi_{\text {Electrode HOMO }}-\varphi_{\mathrm{LUMO}}\right)$. In the case of a bare piezoelectric, $\left(\varphi_{\text {Electrode HOMO }}-\varphi_{\mathrm{LUMO}}\right)$ becomes $\left(\varphi_{\mathrm{VB}}-\varphi_{\mathrm{LUMO}}\right)$. $S_{\min , 2}$ is denoted as the strain necessary to transition from the selective to nonselective regime, e.g. where $\left(\varphi_{\text {Electrode HOMO }}-\varphi_{\text {LUMO }}\right)$ in equation (9) becomes $\left(\varphi_{\text {Electrode HOMO }}-\varphi_{\text {LUMO }}+0.385 \mathrm{~V}\right)$. Using the value parameters fit from experiment in conjunction with the values of piezoelectric materials' parameters listed in Table 1, the $\mathrm{H}_{2}$ generation capacity per straining event $\left(H_{\text {Metal,Total }}\right)$ for a multitude of Au-electrode coated piezoelectric materials is depicted in Figure 3a and is given by a manipulation of equation (6):

$$
\begin{aligned}
H_{\text {Metal,Total }}= & H\left(S-S_{\min , 1}\right) H_{\text {Metal,Smalls }} H\left(S_{\min , 2}-S\right)+ \\
& H\left(S-S_{\text {min }, 2}\right) H_{\text {Metal,LargeS }}
\end{aligned}
$$

$H_{\text {Metal,Smalls }}$ is the $\mathrm{H}_{2}$ production for piezopotentials that fall within the selective regime and is given by:

$$
H_{\text {Metal,Smalls }}=\frac{A C_{e} 2 F D_{H}^{1 / 2} C_{H} f_{2, H} n \sqrt{t_{1}}}{\sqrt{\pi}}
$$

where $t_{1}$ is the time required to exhaust the hydrogen production capability for a piezoelectric operating only within the selective regime $\left(S_{\min , 1}<S<S_{\min , 2}\right)$ and is given by:

$t_{1}=\left(\frac{\left[\frac{\mathrm{V}_{\mathrm{P}_{\text {max }}}}{2}-\left(\varphi_{\text {ElectrodeHOMO }}-\varphi_{\mathrm{LUMO}}\right)\right] \varepsilon_{0} \sqrt{\pi}}{F \frac{n_{H^{+}} w_{x} D_{H^{+}}^{1 / 2} C_{H^{+}} f_{2, H^{+}}}{\varepsilon_{r, x}}+\frac{w_{H}}{\varepsilon_{H_{2 O}}}\left(\sum_{i=m}^{i=1} D_{i}^{1 / 2} C_{i}\left(1-f_{2, i}\right)+\sum_{i=l}^{i=m+1} D_{i}^{1 / 2} C_{i}\right)}\right)$

where $\varepsilon_{\mathrm{H}_{2} \mathrm{O}}$ is the electrical permittivity of water. $\mathrm{H}_{\text {Metal,Larges }}$ is the $\mathrm{H}_{2}$ production for piezopotentials that reach the unbiased regime $\left(S>S_{\min , 2}\right)$ and is given by:
$H_{\text {Metal,LargeS }}=\frac{A C_{e} 2 F D_{H^{+}}^{1 / 2} C_{H^{+}} n_{H^{+}}}{\sqrt{\pi}}\left(f_{1, H^{+}} \sqrt{t_{2}}+f_{2, H^{+}}\left(\sqrt{t_{3}}-\sqrt{t_{2}}\right)\right)$

where $t_{2}$ (equation (14)) is the time it takes for the piezopotential to fall from an arbitrarily large potential $\left(\mathrm{V}_{\mathrm{p}_{\max }}\right)$ to that which brings it into the selective regime $\left(\varphi_{\mathrm{LUMO}}+\varphi_{O p}\right.$, where $\varphi_{O p}$ is the overpotential necessary to transition between reaction regimes, e.g. $0.385 \mathrm{~V}$ ), and $t_{3}$ (equation (15)) is the time it takes for that same piezoelectric to reduce its potential from $\varphi_{\mathrm{LUMO}}+\varphi_{\mathrm{Op}}$ to $\varphi_{\mathrm{LUMO}}$.

$t_{2}=\left(\frac{\left[\frac{\mathrm{V}_{\mathrm{p}_{\text {max }}}}{2}-\left(\left(\varphi_{\text {ElectrodeHOMO }}-\varphi_{\mathrm{LUMO}}\right)+\varphi_{\mathrm{OP}}\right)\right] \varepsilon_{0} \sqrt{\pi}}{4 F \frac{n_{H^{+}} w_{x}}{\varepsilon_{r, x}} \sum_{i=l}^{i=1} D_{i}^{1 / 2} C_{i} f_{1, i}+\frac{w_{H}}{\varepsilon_{H_{2 O}}} \sum_{i=l}^{i=1} D_{i}^{1 / 2} C_{i}\left(1-f_{1, i}\right)}\right)^{2}$

and

$t_{3}=\left(\sqrt{t_{2}}-\frac{\frac{\varphi_{\mathrm{Op}} \varepsilon_{0} \sqrt{\pi}}{2 F}}{\frac{2 n_{H^{+}} w_{x} D_{H}^{1 / 2} C_{H} f_{2, H}}{\varepsilon_{r, x}}+\frac{w_{H}}{\varepsilon_{H_{2 O}}}\left(\sum_{i=m}^{i=1} D_{i}^{1 / 2} C_{i}\left(1-f_{2, i}\right)+\sum_{i=l}^{i=m+1} D_{i}^{1 / 2} C_{i}\right)}\right)_{(15)}^{2}$

At large strains (strain $>$.00025), PZT dominates $\mathrm{H}_{2}$ gas production per unit strain with a value of $9.1 \times 10^{14} \mathrm{H}_{2} \cdot$ strain, PMN-PT and BTO have similar production rates of $5.4 \times 10^{14} \mathrm{H}_{2} \cdot$ strain and $4.5 \times$ $10^{14} \mathrm{H}_{2}$-strain respectively, while $\mathrm{ZnO}$ maintains a modest $\mathrm{H}_{2}$ production rate of $79 \times 10^{13} \mathrm{H}_{2} \cdot$ strain. From equation (13) it can be determined that the $\mathrm{H}_{2}$ production depends upon the relative weight of $f_{1, H^{+}}$to $f_{2, H^{+}}, \mathrm{d}_{x k}$ and $\varepsilon_{r, x}$. When $f_{1, H^{+}}$is much larger than $f_{2, H^{+}}$, $\sqrt{t_{2}}$ dominates the expression where a large $\sqrt{t_{2}}$ value results from a larger $\mathrm{V}_{\mathrm{p}_{\text {max }}}$ combined with a large $\varepsilon_{r, x}$. However $\varepsilon_{r, x}$ is contained within the denominator of the expression determining $\mathrm{V}_{\mathrm{p}_{\max }}$ (equation (1)). Thus, while a large $d_{x k}$ is always valuable to the piezocatalysis process, a balance must be achieved with $\varepsilon_{r, x}$. This is why PZT, possessing both large $\mathrm{d}_{x k}$ and moderately large $\varepsilon_{r, x}$, has the largest $\mathrm{H}_{2}$ production rate. Physically this competition represents a balancing between the piezoelectric, voltage generating capabilities of the material, and the capacitive nature of the material. The inset in Figure 3a enlarges the small strain region (strain $<0.00004$ ), demonstrating the dynamics of initiation $\mathrm{H}_{2}$ production. Materials with the smallest $\frac{\varepsilon_{r, x}}{\mathrm{~d}_{x k}}$ ratio, the most prominent being $\mathrm{ZnO}$, begin $\mathrm{H}_{2}$ production at strains far smaller than a material like PMN-PT. This is because the thermodynamic accessibility of electrochemical reactions depends upon the relative energetics of donor (e $\left.\varphi_{\mathrm{HOMO}}\right)$ and acceptor $\left(e \phi_{L U M O}\right)$ states and a high ratio of piezoelectric moduli to electrical permittivity strongly couples the strain state of the material to these state energetics (equation (9)).

The predicted $\mathrm{H}_{2}$ production capacity is compared to experimental data obtained from an oscillating gold coated PMN-PT cantilever with a peak piezopotential of 20 volts (Fig. 3b) ${ }^{1}$. At $20 \mathrm{~Hz}$, each

\begin{tabular}{|c|c|c|c|c|c|}
\hline Piezoelectric Material & 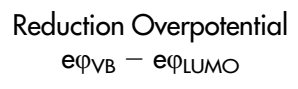 & $\begin{array}{l}\text { Oxidation Overpotential } \\
\qquad е \varphi_{C B}-е \varphi_{\text {номо }}\end{array}$ & BandGap $E_{g}$ & $\varepsilon_{\mathrm{r}, \mathrm{x}}$ & $d_{x k}\left(C N^{-1}\right)$ \\
\hline $\mathrm{ZnO}$ & 3.84 & -0.52 & 3.37 & 8.8 & $11 * 10^{-12}$ \\
\hline PZT & 3.01 & -1.13 & 3.27 & 3850 & $300 * 10^{-12}$ \\
\hline BTO & 2.78 & -1.72 & 3.4 & 1000 & $93 * 10^{-12}$ \\
\hline
\end{tabular}
straining action takes place during 0.025 seconds, which is insufficient for depleting all piezopotential and reaching a thermodynamic

Table 1 | Overpotential and material property values for various piezoelectric materials ${ }^{12,19,21-26}$ 

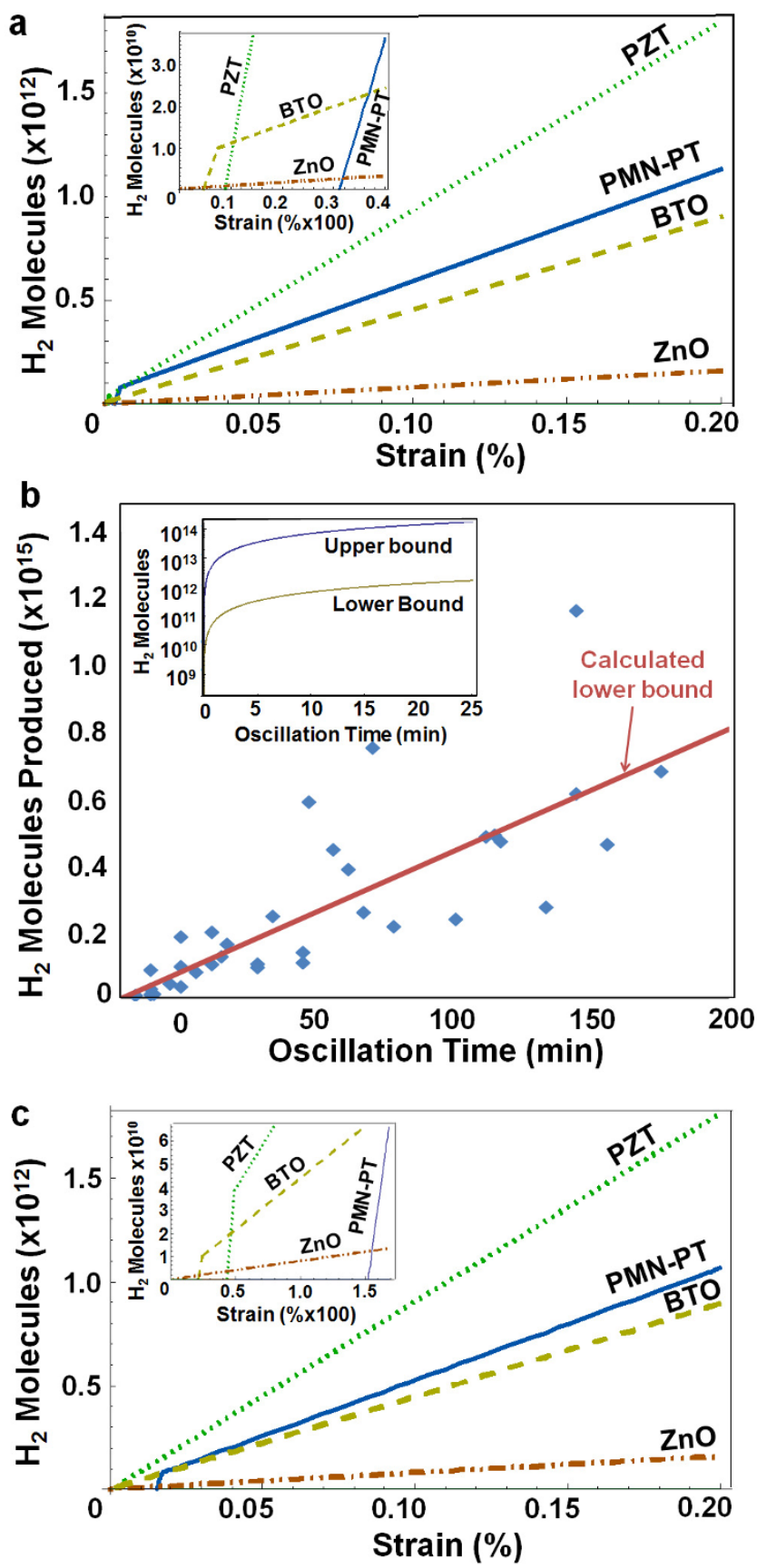

Figure $3 \mid$ The $\mathrm{H}_{2}$ production per straining event predicted as a function of strain magnitude. (a) In the case of piezoelectric materials with metal electrodes on their surfaces. Inset shows the turn-on strain value is dependent on the ratio of $\frac{\varepsilon_{r, x}}{\mathrm{~d}_{x k}}$. (b) Comparison between experimental data of hydrogen generation over the course of an oscillating PMN-PT cantilever experiment and the predicted results from simulation under the conditions of poor kinetics $\left(f_{\mathrm{i}}=0.07\right)$. Inset shows the envelope of hydrogen generation predicted from simulation under the case of good kinetics $\left(f_{\mathrm{i}}=0.715\right)$ and poor kinetics. (c) In the case of bare surfaced piezoelectric materials. Inset shows the turn-on strain values depends upon both the ratio of $\frac{\varepsilon_{r, x}}{\mathrm{~d}_{x k}}$ and the value of $\varphi_{O p}$.

equilibrium. Nonetheless, an approximation of the experiment can be constructed by truncating the nonselective regime at 0.025 seconds and applying a window of acceptable values on the kinetics parameter $f_{\mathrm{i}}$ from 0.07 to 0.715 (inset of Fig. $3 \mathrm{~b}$ ). Experimental data fall around the lower bound of predicted $\mathrm{H}_{2}$ evolution rate, exhibiting good agreement. This indicates that there remains much room for experimentally improving the $\mathrm{H}_{2}$ production rate by means of adding a proton reduction co-catalyst and thus increasing the $f_{\mathrm{i}}$ value.

Several factors change when comparing a piezoelectric material coated with metal electrodes to a naked, insulating one. In general, a naked and perfectly insulating piezoelectric material does not necessarily possess a continuum of states about the redox potentials in solution. To achieve piezocatalysis (including $\mathrm{H}_{2}$ production) with such a piezoelectric material, the conduction band and valence band must be moved sufficiently in potential so that their energies coincide with redox potentials under examination. Taking the production of $\mathrm{H}_{2}$ as our prototypical case, the energetics of top-most valence band electrons $\left(\mathrm{e} \varphi_{\mathrm{VB}}\right)$ must be lifted above the $\left(\mathrm{e} \phi_{L U M O}\right)$ of $\mathrm{H}_{2}$. The minimum strain, and thus potential, required to achieve this criteria is again given by equation (9), except in the case of a bare piezoelectric $\varphi_{\text {ElectrodeHOMO becomes }} \varphi_{\mathrm{VB}}$. The values of potentials necessary to cause the reduction of $\mathrm{H}^{+}$and oxidation of $\mathrm{H}_{2} \mathrm{O}$ for various piezoelectric materials can be found in Table 1 . The hydrogen production capacity of a bare piezoelectric material can be calculated by modifying equation (10):

$$
\begin{aligned}
H_{\text {Insulator,Total }}= & H\left(S_{\min , 1}-S\right) H_{\text {Insulator,Smalls }} H\left(S-S_{\min , 2}\right)+ \\
& H\left(S-S_{\min , 2}\right) H_{\text {Insulator,LargeS }}
\end{aligned}
$$

where $H_{\text {Insulator,Smalls }}$ is the $\mathrm{H}_{2}$ production for piezopotentials that fall within the selective regime $\left(S_{\min , 1}<S<S_{\min , 2}\right), H_{\text {Insulator,Larges }}$ is the $\mathrm{H}_{2}$ production for piezopotentials capable to reaching the nonselective regime $\left(S>S_{\min , 2}\right)$.

Applying the values from Table 1 and the same kinetic parameters as in the metal electrode case $\left(f_{1 \mathrm{i}}=0.715, f_{2 \mathrm{i}}=0.07\right.$, and $\varphi_{O p}=$ $-0.385 \mathrm{~V})$, Fig. $3 \mathrm{c}$ shows the $\mathrm{H}_{2}$ production rate for various naked piezoelectric materials. Subjected to large strains (strain $>.001$ ), both naked and Au coated piezoelectric materials perform consonantly. Discrepancies between these two are more prominent under small strain (strain $<0.0002$ ), which are a result of the difference in the band structures of individual piezoelectric materials. Under small strain, each piezoelectric's $\mathrm{H}_{2}$-production's turn-on strain depends not only upon the $\frac{\varepsilon_{r, x}}{\mathrm{~d}_{x k}}$ ratio but also upon the value of the individual piezoelectric material's $\left(\mathrm{e} \varphi_{\mathrm{VB}}-\mathrm{e} \varphi_{\mathrm{LUMO}}\right)$ value.

Concerns over the stability of a piezocatalysis system comprising a bare piezoelectric material can be addressed by comparing piezocatalysis with a controlled corrosion process, where reduction reactions take place through the piezoelectric's oxidation and vice versa. The maximum piezoelectric charge density at the surface is on the order of $10^{13}$ charge $\mathrm{cm}^{2}$, two orders of magnitude lower than the average atomic density of a solid-state surface. With oxidation events of this magnitude, it is expected that a piezoelectric capable of withstanding photocatalytic reactions with water is also able to survive the piezocatalysis process.

In contrast to the previous analysis, most piezoelectric materials are not perfect insulators, mobile electrical charges inside the piezoelectric material respond to and rearrange according to its internal electric field. In bulk systems, these mobile charges act as extremely effective screening agents of the piezopotential, resulting in an effective piezopotential given by a manipulation of the Gouy-Chapman capacitance model:

$$
V_{\text {Semi }}=\frac{2 k T \sinh ^{-1}\left[d_{x k} S_{k} Y \sqrt{\varepsilon_{0} \varepsilon_{r, x} k n_{0} T}\right]}{2 \sqrt{2} \varepsilon_{0} \varepsilon_{r, x} k n_{0} T}
$$

where $k$ is Boltzmann's constant, and $n_{0}$ is the bulk concentration of free charge $\mathrm{e}^{17}$. According to equation (17), a highly insulating bulk piezoelectric is required to reach an appreciable potential (Fig. 4a). Taking the values from Table 1 for various piezoelectric materials subjected to a strain of 0.002 , the maximum concentration of free charges allowed in order for the piezoelectric materials' surfaces to obtain the $\mathrm{H}_{2}$ production potential were $5.22 \times 10^{-40}, 2.33 \times 10^{-27}$, $9.37 \times 10^{-23}$, and $1.38 \times 10^{-26} \mathrm{~m}^{-3}$, for ZNO, PMNPT, BTO and 

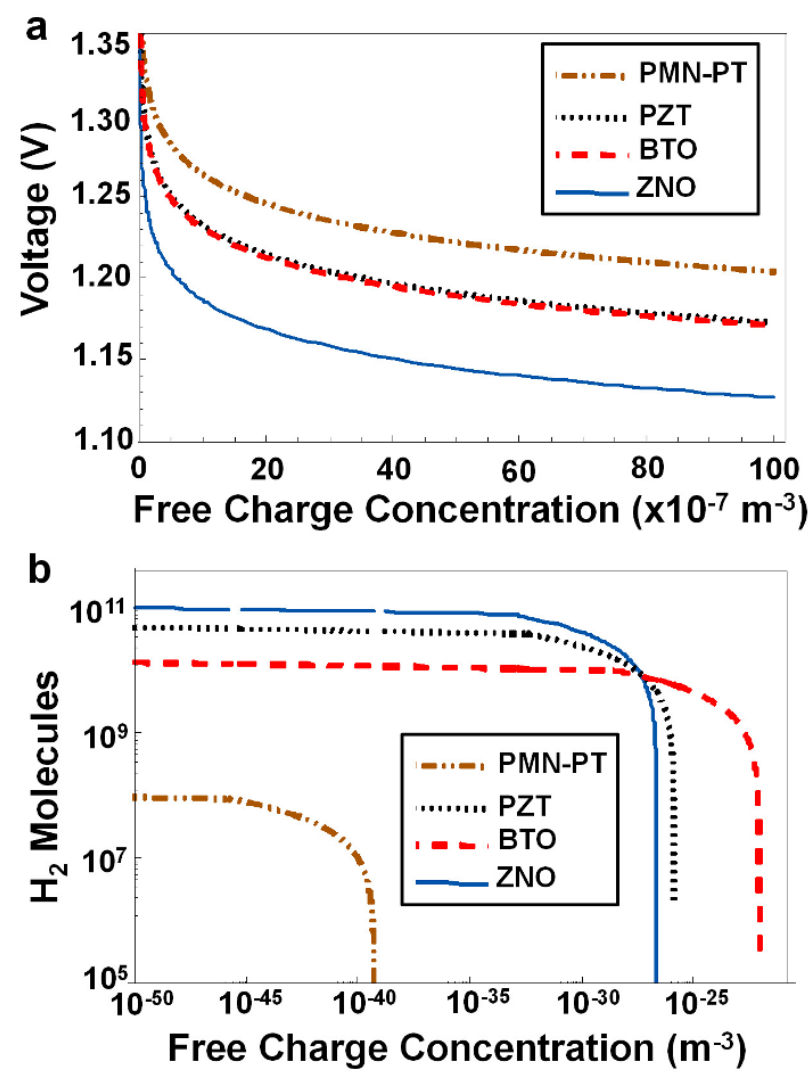

Figure 4 Free charge can dramatically affect the piezocatalytic ability of a strained piezoelectric. For a strain $=.0 .002$, the effect of free charge on (a) piezoelectric potential and (b) $\mathrm{H}_{2}$ production for various piezocatalysis systems.

PZT respectively. The $\mathrm{H}_{2}$ production capacity for these piezoelectric materials subjected to $0.2 \%$ strain was determined as a function of free charge by manipulating equation (16):

$$
\begin{aligned}
H_{\text {Semi }, \text { Total }}= & H\left(n_{\max , 1}-n_{0}\right) H_{\text {Highn }_{0}} H\left(n_{0}-n_{\max , 2}\right)+ \\
& H\left(n_{0}-n_{\max , 2}\right) H_{\text {Lown }_{0}}
\end{aligned}
$$

where $H_{H i g h n_{0}}$ is the expression for $\mathrm{H}_{2}$ production while the piezopotential is within the selective regime and $H_{\text {Lown }}$ describes the $\mathrm{H}_{2}$ production for a piezoelectric that has achieved a potential for accessing the nonselective regime. $n_{\max , 1}$ is the maximum number of free charges allowed for a given piezoelectric material under $0.2 \%$ strain to reach the threshold potential $\left(\varphi_{\mathrm{VB}}-\varphi_{\mathrm{LUMO}}\right)$ for driving $\mathrm{H}_{2}$ production. $n_{\max , 2}$ is the charge concentration necessary to reach the potential threshold between the two reaction regimes $\left[\left(\varphi_{\mathrm{VB}}-\varphi_{\mathrm{LUMO}}\right)+\varphi_{\mathrm{OP}}\right]$. In equation (18) the dependence of $\mathrm{H}_{2}$ production on $n_{0}$ comes exclusively from the fact that in the case of a semiconducting piezoelectric material, $V_{p, \max }$ [equation (13) \& (14)] becomes $V_{\text {Semi }}$ [equation (17)].

The effect of free charge on $\mathrm{H}_{2}$ production for various piezoelectric materials is demonstrated in Fig. 4b, where the hydrogen production for a strain of $0.2 \%$ is shown as a function of mobile charge concentration, $n_{0}$. Behavior of particular note is the dramatic increase in $\mathrm{H}_{2}$ production that results from decreasing the mobile charge concentration $\sim 5$ orders of magnitude below the minimum concentration required to reach the $\mathrm{H}_{2}$ potential for the piezoelectric materials. Additional mobile charge reduction beyond that initial 5 orders reduction has relatively little effect on $\mathrm{H}_{2}$ evolution.

\section{Discussion}

A number of factors have been shown to augment the efficacy of strain to induce electrochemical reactions. The relative energies of states within the electrode with respect to HOMO and LUMO energies in solution can both dramatically change the rate of chemical reactions and be a determining factor in which chemical reactions are allowed to proceed. These energy state positions depend directly on the magnitude of strain and piezoelectric coefficient while depending inversely upon the electrical permittivity. In the presence of freecharge, generated either by doping, photo or thermal excitations, the piezopotential can be markedly decreased with direct repercussions on reactivity. In order to increase the electrochemical activity of a strained piezoelectric, the value electrical permittivity needs to be optimized. The piezoelectric, semiconductor and molecular orbital frameworks discussed herein have historically and successfully been applied to solid state and electrochemical systems. It is expected that these frameworks can be extended to the piezocatalysis theory. The theoretical work presented here can provide fundamental guidance for designing and understanding the novel piezocatalysis phenomenon from broad electrochemistry and piezoelectric material systems.

\section{Methods}

All calculations were performed in Wolfram Mathematica 8. To calculate the total $\mathrm{H}_{2}$ output from an insulating piezoelectric with metal electrodes, we first calculated the $\mathrm{H}_{2}$ generated as the piezoelectric potential dropped from the maximum potential induced by strain [equation(1)] to the potential present at time $t_{\mathrm{p}}$. Then, the $\mathrm{H}_{2}$ generated after time $t_{\mathrm{p}}$ was calculated using another value of the kinetic parameter. Summation of these two $\mathrm{H}_{2}$ quantities gave the total $\mathrm{H}_{2}$ output. The first time segment utilized kinetics parameter $f_{1}=0.715$; while the second segment utilized $f_{2}=0.07$. To accomplish this we used equation (10), using the variable values found within the paper. Species $i=1, \ldots m$ was designated as $\mathrm{H}^{+}$ions only, while $i=m+1, \ldots l$ included all other species in solution.

Calculations conducted on bare, insulating piezoelectric materials used the same methodology as those done for metal electrodes-covered insulating piezoelectric materials. The only difference is that the valence band and conduction band energies of the piezoelectric material were used in place of the metal's Fermi energy (equation 16 and Table 1). In the case of the semiconducting piezoelectric, the same procedure was followed the case of the insulating piezoelectric [equation (18)] except that $\mathrm{V}_{\text {Semi }}$ [equation (17)) was used in place of $\mathrm{V}_{\mathrm{P} \text { max }}$.

The experimental data was acquired by using a PMN-PT single crystal slab as the piezoelectric component for piezocatalyzed water splitting (from our previously publication $\left.^{1}\right)$. The piezoelectric cantilever architecture was constructed and placed within a sealed chamber with access ports for piezoelectric actuation and monitoring, environmental purging, and atmospheric sampling. Straining of the piezoelectric cantilever was achieved by external electronic actuators. The $\mathrm{H}_{2}$ concentration was determined by an AMETEK Analyzer ta3000F $\mathrm{H}_{2}$ gas analyzer. The piezoelectric potential was measured by a digital oscilloscope and a potentiostat, respectively. See reference 1 for more details.

1. Starr, M. B., Shi, J. \& Wang, X. D. Piezopotential-Driven Redox Reactions at the Surface of Piezoelectric Materials. Angewandte Chemie-International Edition 51, 5962-5966 (2012).

2. Mantini, G., Gao, Y., D’Amico, A., Falconi, C. \& Wang, Z. Equilibrium piezoelectric potential distribution in a deformed $\mathrm{ZnO}$ nanowire. Nano Research 2, 624-629 (2009).

3. Hamann, T. W., Gstrein, F., Brunschwig, B. S. \& Lewis, N. S. Measurement of the free-energy dependence of interfacial charge-transfer rate constants using $\mathrm{ZnO} /$ $\mathrm{H} 2 \mathrm{O}$ semiconductor/liquid contacts. Journal of the American Chemical Society 127, 7815-7824 (2005).

4. Hamann, T. W., Gstrein, F., Brunschwig, B. S. \& Lewis, N. S. Measurement of the dependence of interfacial charge-transfer rate constants on the reorganization energy of redox species at $\mathrm{n}-\mathrm{ZnO} / \mathrm{H} 2 \mathrm{O}$ interfaces. Journal of the American Chemical Society 127, 13949-13954 (2005).

5. Hamann, T. W., Gstrein, F., Brunschwig, B. S. \& Lewis, N. S. Measurement of the driving force dependence of interfacial charge-transfer rate constants in response to $\mathrm{pH}$ changes at n-ZnO/H2O interfaces. Chemical Physics 326, 15-23 (2006).

6. Hamann, T. W., Gstrein, F. \& Lewis, N. S. Driving force dependence of electron transfer rate constants at the $\mathrm{ZnO}$-liquid interface. Abstracts of Papers of the American Chemical Society 226, U655-U655 (2003).

7. Xue, X. Y., Wang, S. H., Guo, W. X., Zhang, Y. \& Wang, Z. L. Hybridizing Energy Conversion and Storage in a Mechanical-to-Electrochemical Process for SelfCharging Power Cell. Nano Lett. 12, 5048-5054 (2012). 
8. Kalinin, S. V., Bonnell, D. A., Alvarez, T., Lei, X., Hu, Z., Shao, R. \& Ferris, J. H. Ferroelectric lithography of multicomponent nanostructures. Adv. Mater. 16, 795-799 (2004).

9. Li, D. B. \& Bonnell, D. A. Ferroelectric lithography. Ceram. Int. 34, 157-164 (2008).

10. Bhardwaj, A., Burbure, N. V., Gamalski, A. \& Rohrer, G. S. Composition Dependence of the Photochemical reduction of Ag by Bal-xSrxTiO3. Chemistry of Materials 22, 3527-3534 (2010).

11. Burbure, N. V., Salvador, P. A. \& Rohrer, G. S. Photochemical Reactivity of Titania Films on BaTiO3 Substrates: Origin of Spatial Selectivity. Chemistry of Materials 22, 5823-5830 (2010).

12. Burbure, N. V., Salvador, P. A. \& Rohrer, G. S. Photochemical Reactivity of Titania Films on BaTiO3 Substrates: Influence of Titania Phase and Orientation. Chemistry of Materials 22, 5831-5837 (2010).

13. Jones, P. M., Gallardo, D. E. \& Dunn, S. Photochemical investigation of a polarizable semiconductor, lead-zirconate-titanate. Chemistry of Materials 20, 5901-5906 (2008).

14. Bhardwaj, A., Burbure, N. V. \& Rohrer, G. S. Enhanced Photochemical Reactivity at the Ferroelectric Phase Transition in Ba1-xSrxTiO3. Journal of the American Ceramic Society 93, 4129-4134 (2010).

15. Burbure, N. V., Salvador, P. A. \& Rohrer, G. S. Orientation and Phase Relationships between Titania Films and Polycrystalline BaTiO3 Substrates as Determined by Electron Backscatter Diffraction Mapping. Journal of the American Ceramic Society 93, 2530-2533 (2010).

16. Wang, X. Piezoelectric nanogenerators-Harvesting ambient mechanical energy at the nanometer scale. Nano Energy 1, 13-24 (2012).

17. A. J. Bard, L. R. F. Electrochemical Methods: Fundamentals and Applications, Edn. second. (Wiley, New York; 2001).

18. Pletcher, D. A First Course in Electrode Processes. (Royal Society of Chemistry, Cambridge; 2009.).

19. Jue, P., Haosu, L., He, T., Haiqing, X. \& Lin, D. Elastic, dielectric, and piezoelectric characterization of $0.70 \mathrm{~Pb}(\mathrm{Mg} 1 / 3 \mathrm{Nb} 2 / 3) \mathrm{O} 3-0.30 \mathrm{PbTiO} 3$ single crystals. Materials Letters 59, 640-643 (2005).

20. Millipore, E. Billerica (2013).
21. PiezoTechnologies Indianapolis (2013).

22. Look, D. C. et al. Electrical properties of bulk ZnO. Solid State Communications 105, 399-401 (1998).

23. Coleman, V. A. \& Jagadish, C. Basic Properties and Applications of ZnO. (2006).

24. Chen, X., Shen, S., Guo, L. \& Mao, S. S. Semiconductor-based Photocatalytic Hydrogen Generation. Chemical Reviews 110, 6503-6570 (2010).

25. Chen, C. Q., Shi, Y., Zhang, Y. S., Zhu, J. \& Yan, Y. J. Size Dependence of Young's Modulus in ZnO Nanowires. Physical Review Letters 96, 075505 (2006).

26. Bak, T., Nowotny, J., Rekas, M. \& Sorrell, C. C. Photo-electrochemical hydrogen generation from water using solar energy. Materials-related aspects. International Journal of Hydrogen Energy 27, 991-1022 (2002).

\section{Acknowledgments}

This work is supported by DARPA under grant No. N66001-11-1-4139 and National Science Foundation CMMI-1148919.

\section{Author contributions}

M.S. performed the calculations. M.S. and X.W. analyzed the data and interpreted the results. M.S. and X.W. wrote the manuscript.

\section{Additional information}

Competing financial interests: The authors declare no competing financial interests.

How to cite this article: Starr, M.B. \& Wang, X. Fundamental Analysis of Piezocatalysis Process on the Surfaces of Strained Piezoelectric Materials. Sci. Rep. 3, 2160; DOI:10.1038/ srep02160 (2013)

\footnotetext{
cc)(1) (2) This work is licensed under a Creative Commons Attribution-

Co NC SA NonCommercial-ShareAlike 3.0 Unported license. To view a copy of this license,
} visit http://creativecommons.org/licenses/by-nc-sa/3.0 